\title{
Synthesis and Characterization of Impurities of an Broad- Spectrum Anthelmintic Drug, Albendazole
}

\author{
SABRINTAJ KALAS, SURYAKANT PATIL ${ }^{*}$ and VIDYADHAR JADHAV
}

Prof. Dr. S. C. Bhattacharya Organic Synthesis Laboratory, VerGo Pharma Research Lab. Pvt. Ltd., Plot B5, Phase 1A, Verna Industrial Estate, Verna, Salcette, Goa-403 722, India suryakant.patil@vergophama.com

Recived 10 October 2015 / Accepted 28 October 2015

\begin{abstract}
Albendazole (1) is a broad-spectrum anthelmintics drug. Albendazole impurity A, 5(propylthio)- $1 H$-benzimidazol-2-amine (6), impurity B, methyl [5-(propylsulfinyl)- $1 H$-benzimidazol2-yl]carbamate (7), impurity C, methyl [5-(propylsulfonyl)-1 $H$ - benzimidazol-2-yl]carbamate $(\mathbf{8})$ and impurity D, 5-(propylsulfonyl)- $1 H$ - benzimidazol-2-amine (9) are degradation or metabolic impurities whereas impurity E, methyl $1 H$-benzoimidazol- 2-yl)carbamate (11) and impurity F, methyl [5(methylthio)- $1 \mathrm{H}$ - benzimidazol-2-yl]carbamate (15) are process related impurities. All these impurities are listed in European pharmacopoeia. Present work describes the synthesis and characterization of all these six impurities.
\end{abstract}

Keywords: Albendazole, Impurities, Synthesis, Characterization

\section{Introduction}

Albendazole is a broad-spectrum anthelmintics against gastrointestinal nematodes and the larval stages of cestodes ${ }^{1}$. Recently, the anticancer effects of albendazole were also investigated $^{2,3}$. The principal mode of action for Albendazole is by its inhibitory effect on tubulin polymerization which results in the loss of cytoplasmic microtubules. Albendazole was first discovered at the SmithKline Animal Health Laboratories in 1972.

Impurities can be closely related to the product that is formed during the synthesis of a bulk drug or it can be decomposition product formed during the storage of the drug. The presence of impurities in an active pharmaceutical ingredient (API) can have a significant impact on the quality and safety of the drug products. Therefore, it is necessary to study the impurity profile of the API to be used in the manufacturing of drug substance. International conference on harmonization (ICH) guidelines recommended identifying and characterizing all impurities that are present at a level of $0.10 \%$ or more ${ }^{4,5}$.

Albendazole is from benzimidazole carbamate family chemically known as methyl-[(6propylthio)-1H-benzimidazol-2-yl]carbamate (1). Albendazole synthesis using various methods 
is already disclosed ${ }^{6,7}$. Recently efficient process for albendazole preparation at scale is reported $^{8}$. Various albendazole analogues were synthesized and evaluated for their biological activity ${ }^{9,10}$.

\section{Experimental}

The IR spectra were recorded in the solid state as using Shimadzu IR Affinity-1 spectrophotometer. ${ }^{1} \mathrm{H}$ NMR spectra were recorded on Bruker Advance III $400 \mathrm{MHz}$ spectrometer, the chemical shifts are reported in $\delta$ ppm relative to TMS. The mass spectra were recorded on LCMS- API-2000, ABSciex. Albendazole API was synthesized as per literature method ${ }^{8}$. Reaction conditions mentioned here are not optimized and yields are of isolated and purified products.

\section{5-(Propylthio)-1H-benzimidazol-2-amine (6)}

Reaction mixture consisting solution of albendazole $(1,3.0 \mathrm{~g}, 11.3 \mathrm{mmole})$ in concentrated sulphuric acid: water $(30 \mathrm{~mL}, 1: 1 ; \mathrm{v} / \mathrm{v})$ was heated to reflux temperature $\left(100{ }^{\circ} \mathrm{C}\right)$ maintained while stirring for $16 \mathrm{~h}$ at same temperature. Reaction progress was monitored by TLC ( $100 \%$, ethyl acetate, $\left.\mathrm{R}_{f}=0.1\right)$. Reaction mass was then cooled to room temperature $\left(23-25{ }^{\circ} \mathrm{C}\right)$ and poured into ice cold water $(100 \mathrm{~mL})$. Reaction mass was basified using aqueous sodium hydroxide solution $(10 \%, 200 \mathrm{~mL})$. Reaction mixture was extracted using ethyl acetate $(3 \times 50 \mathrm{~mL})$, organic layers were combined dried over sodium sulfate, filtered and concentrated under reduced pressure to get crude product residue. Crude product residue was purified by silica gel column chromatography to get buff colored solid, yield $28 \%, 0.65 \mathrm{~g}$, HPLC purity $89.80 \%, \mathrm{mp} 154.6{ }^{\circ} \mathrm{C}$; IR $\left(\mathrm{V}_{\max }, \mathrm{cm}^{-1}\right) 3388(\mathrm{~N}-\mathrm{H}), 2960-2872$ (aliphatic-H), 1651-1645 (C=C stretching), 1556 (C-C stretching ring), 1016, 885, 748 (C-H bending). ${ }^{1} \mathrm{H}$ NMR (DMSO-d $\left.{ }_{6}\right) 10.69(1 \mathrm{H}, \mathrm{bs}, \mathrm{NH}), 7.05-7.048\left(1 \mathrm{H}_{\text {arom }}, \mathrm{d}\right), 6.97-6.94\left(1 \mathrm{H}_{\text {arom }}, \mathrm{d}\right), 6.85-$ $6.81\left(1 \mathrm{H}_{\text {arom }}, \mathrm{dd}\right), 6.2\left(2 \mathrm{H}\right.$, bs, $\left.\mathrm{NH}_{2}\right), 2.75-2.65\left(2 \mathrm{H}, \mathrm{t},-\mathrm{CH}_{2}\right), 1.46-1.39(2 \mathrm{H}, \mathrm{q}$, $\left.\mathrm{CH}_{2} \mathrm{CH}_{2} \mathrm{CH}_{3}\right), 0.90-0.84\left(3 \mathrm{H}, \mathrm{t},-\mathrm{CH}_{3}\right)$. MS, $m / z 208[\mathrm{M}+\mathrm{H}]^{+}$.

\section{Methyl [5-(propylsulfinyl)-1H-benzimidazol-2-yl]carbamate (7)}

To a solution of Albendazole $(1,3.0 \mathrm{~g}, 11.3 \mathrm{mmole})$ in methanol: water $(30 \mathrm{~mL} ; 1: 1$; v/v) was added oxone $(1.72 \mathrm{~g} ; 11.3 \mathrm{mmole})$ in portions at $5-10{ }^{\circ} \mathrm{C}$. Reaction mass was then warmed to room temperature, stirred for $14 \mathrm{~h}$. Solid obtained was filtered, washed with water $(20 \mathrm{~mL})$. Filtrate wasconcentrated under reduced pressure to get crude product was dissolved in methanol in dichloromethane $(20 \mathrm{~mL}, 1: 9 ; \mathrm{v} / \mathrm{v})$. Solid formation was observed was filtered and filtrate was concentrated under reduced pressure to get off-white colored solid, yield 28\%, $0.90 \mathrm{~g}$, HPLC purity $95.35 \%, \mathrm{mp} 195.1^{\circ} \mathrm{C}$; IR $\left(\mathrm{V}_{\max }, \mathrm{cm}^{-1}\right) 3223(\mathrm{~N}-\mathrm{H})$, 2958-2866 (aliphatic-H), 1755 (amide N-H), 1651-1633 (C=C stretching), 1467 (C-H stretching), 1016, 885, 748 (C-H bending). ${ }^{1} \mathrm{H}-\mathrm{NMR}\left(\mathrm{DMSO}_{6}\right) 7.8\left(1 \mathrm{H}_{\text {arom }}, \mathrm{s}\right), 7.7\left(1 \mathrm{H}_{\text {arom }}\right.$, d), $7.5\left(1 \mathrm{H}_{\text {arom }}, \mathrm{d}\right), 3.9\left(3 \mathrm{H}, \mathrm{s},-\mathrm{OCH}_{3}\right), 3.7-3.8\left(2 \mathrm{H}, \mathrm{t},-\mathrm{SCH}_{2}\right), 1.5-1.6\left(2 \mathrm{H}, \mathrm{m},-\mathrm{CCH}_{2}\right), 0.9$ $\left(3 \mathrm{H}, \mathrm{t},-\mathrm{CH}_{3}\right)$. MS, $m / z 282[\mathrm{M}+\mathrm{H}]^{+}$.

\section{Methyl [5-(propylsulfonyl)-1H-benzimidazol-2-yl]carbamate (8)}

To a solution of Albendazole $(\mathbf{1}, 6.0 \mathrm{~g}, 22.6 \mathrm{mmole})$ in dichloromethane $(100 \mathrm{~mL})$ was added $m$-chloroperbenzoic acid $(7.8 \mathrm{~g}, 45.3 \mathrm{mmole})$ in portions at $5-10{ }^{\circ} \mathrm{C}$. Reaction mass was then warmed to room temperature $\left(23-25{ }^{\circ} \mathrm{C}\right)$, stirred for $9 \mathrm{~h}$. Reaction progress was monitored by TLC (petroleum ether:ethyl acetate, 1:1, $\mathrm{R}_{f}=0.12$ ). Reaction mass was concentrated under reduced pressure to get residue as crude product. Crude product was purified on silica gel column chromatography to get off-white solid, yield 97\%, $6.50 \mathrm{~g}$, HPLC 
purity $93.94 \%, \mathrm{mp} 282.6{ }^{\circ} \mathrm{C}$; IR $\left(\mathrm{v}_{\max }, \mathrm{cm}^{-1}\right) 3234(\mathrm{~N}-\mathrm{H}), 2958-2866$ (aliphatic-H), 1697 (amide $\mathrm{N}-\mathrm{H}$ ), 1651-1643 (C=C stretching), 1595-1427 (stretching in ring), 1271-1107 (SO stretching). ${ }^{1} \mathrm{H}$ NMR (DMSO-d $\left.\mathrm{d}_{6}\right) 12.35(1 \mathrm{H}, \mathrm{bs},-\mathrm{NH}), 11.64(1 \mathrm{H}, \mathrm{bs},-\mathrm{NH}), 7.9\left(1 \mathrm{H}_{\text {arom }}\right.$, s), $7.6\left(2 \mathrm{H}_{\text {arom }}, \mathrm{dd}\right), 3.8\left(3 \mathrm{H}, \mathrm{s},-\mathrm{OCH}_{3}\right), 3.25\left(2 \mathrm{H}, \mathrm{t},-\mathrm{SCH}_{2}\right), 1.5\left(2 \mathrm{H}, \mathrm{m},-\mathrm{CCH}_{2}\right), 0.85$ $\left(3 \mathrm{H}, \mathrm{t},-\mathrm{CH}_{3}\right) . \mathrm{MS}, \mathrm{m} / z 298[\mathrm{M}+\mathrm{H}]^{+}$.

\section{5-(Propylsulphonyl)-1H-benzimidazol-2-amine (9)}

Reaction mixture consisting solution of $(\mathbf{8})(2.5 \mathrm{~g}, 8.4 \mathrm{mmole})$ in concentrated sulphuric acid: water $(30 \mathrm{~mL}, 1: 1 ; \mathrm{v} / \mathrm{v})$ was heated to reflux temperature $\left(100{ }^{\circ} \mathrm{C}\right)$ maintain while stirring for $13 \mathrm{~h}$ at same temperature. Reaction progress was monitored by TLC (100\%, ethyl acetate, $\mathrm{R}_{f}=0.13$ ). Reaction mass was cooled to room temperature and poured on icecold water $(100 \mathrm{~mL})$. Reaction mass was washed with ethyl acetate $(2 \times 50 \mathrm{~mL})$ to remove non-polar impurities. Reaction mass was basified using aqueous sodium hydroxide solution $(10 \%, 200 \mathrm{~mL})$ and extracted using ethyl acetate $(3 \times 50 \mathrm{~mL})$, organic layers were combined dried over sodium sulfate, filtered and concentrated under reduced pressure to get orange colored solid (hygroscopic), yield 39\%, $0.78 \mathrm{~g}$, HPLC purity 96.68\%; IR $\left(\mathrm{v}_{\max }, \mathrm{cm}^{-1}\right) 3344$ (N-H), 2958-2866 (aliphatic-H), 1651-1643 (N-H), 1427-1276 (SO stretching). ${ }^{1} \mathrm{H}$ NMR (DMSO-d $\left.{ }_{6}\right) 11.2(1 \mathrm{H}, \mathrm{bs}$, indole- $\mathrm{NH}), 7.5\left(1 \mathrm{H}_{\text {arom }}, \mathrm{s}\right), 7.35\left(1 \mathrm{H}_{\text {arom }}, \mathrm{d}\right), 7.25\left(1 \mathrm{H}_{\text {arom }}, \mathrm{d}\right), 6.7$ $\left(2 \mathrm{H}, \mathrm{s}, \mathrm{NH}_{2}\right), 3.15\left(2 \mathrm{H}, \mathrm{t},-\mathrm{SCH}_{2}\right), 1.5\left(2 \mathrm{H}, \mathrm{m},-\mathrm{CCH}_{2}\right), 0.8-0.9\left(3 \mathrm{H}, \mathrm{t},-\mathrm{CH}_{3}\right) . \mathrm{MS}, \mathrm{m} / \mathrm{z} 240$ $[\mathrm{M}+\mathrm{H}]^{+}, 262[\mathrm{M}+\mathrm{Na}]$.

\section{Methyl 1H-benzoimidazol-2-yl carbamate (11)}

To a solution of 2-aminobenzimidazole (10) $(2.0 \mathrm{~g}, 15.0 \mathrm{mmole})$ in acetone $(30 \mathrm{~mL})$ was added triethylamine (1.8 g, 18.0 mmole) at room temperature, stirred for $15 \mathrm{~min}$. To above reaction mass was added methyl chloroformate $(1.7 \mathrm{~g}, 17.0 \mathrm{mmole})$ drop wise at room temperature, reaction mass was stirred for $10 \mathrm{~h}$ at same temperature. Reaction was monitored by TLC ( $50 \%$ ethyl acetate in petroleum ether, $\mathrm{R} f=0.65)$. Solid appeared was filtered off, washed with little acetone $(50 \mathrm{~mL})$ then with water $(20 \mathrm{~mL})$. Solid dried under vacuum to get white colored solid, yield $35 \%, 1.50 \mathrm{~g}$, HPLC purity $99.59 \%$, mp $168.2{ }^{\circ} \mathrm{C}$; IR $\left(\mathrm{V}_{\max }, \mathrm{cm}^{-1}\right) 3442(\mathrm{~N}-\mathrm{H}), 2601-2497$ (aliphatic-H), 1722 (amide $\left.\mathrm{N}-\mathrm{H}\right), 1647(\mathrm{C}=\mathrm{C}$ stretching), 1456, 1367 (aliphatic-H bending), 1215 (C-O-C asymmetric stretching), 765, 748 (C-H bending). ${ }^{1} \mathrm{H}$ NMR (DMSO-d $\left.\mathrm{d}_{6}\right)$ : 7.95-7.90 ( $\left.1 \mathrm{H}_{\text {arom }}, \mathrm{m}\right), 7.15-7.04\left(2 \mathrm{H}_{\text {arom }}, \mathrm{m}\right), 7.18$ $\left(1 \mathrm{H}, \mathrm{s}\right.$, indole-NH), $7.55\left(1 \mathrm{H}_{\text {arom }}, \mathrm{d}\right), 3.95\left(3 \mathrm{H}, \mathrm{s},-\mathrm{OCH}_{3}\right)$. MS, $\mathrm{m} / \mathrm{z} 192[\mathrm{M}+\mathrm{H}]^{+}$.

\section{Methyl [5-(methylthio)-1H-benzimidazol-2-yl]carbamate(15)}

\section{Step-I: 2-Nitro-4-thiocyanoaniline (3)}

To a mixture of 2-nitroaniline (5.0 g, 36.2 mmole) and ammonium thiocyanate $(5.65 \mathrm{~g}, 74.2$ mmole) in methanol $(40 \mathrm{~mL})$ was purged chlorine gas (generated in laboratory using potassium permanganate and hydrochloric acid) for $6 \mathrm{~h}$ at room temperature, stirred for $2 \mathrm{~h}$ at same temperature. Reaction progress was monitored by TLC (20\% ethyl acetate in petroleum ether, $\left.\mathrm{R}_{f}=0.4\right)$. To reaction mass was added water $(50 \mathrm{~mL})$, stirred at $20^{\circ} \mathrm{C}$ for $1 \mathrm{~h}$. Solid was filtered, washed with water $(20 \mathrm{~mL})$ and dried under vacuum to give yellow colored solid (3), yield 59\%, $4.2 \mathrm{~g}$. This was used as such for the next step.

\section{Step-II: 4-(Methylthio)-2-nitroaniline (12)}

To a solution of (3) (4.0 g, 20.5 mmole) in methanol:water $(20 \mathrm{~mL}, 1: 1$, v/v) was added sodium hydroxide ( $3.44 \mathrm{~g}, 86.0 \mathrm{mmole})$ at room temperature. Reaction mass then heated to $40{ }^{\circ} \mathrm{C}$, stirred at same temperature for $1 \mathrm{~h}$. Reaction mass was cooled to $10-15{ }^{\circ} \mathrm{C}$ was added 
methyl iodide ( $2.61 \mathrm{~mL}, 41.0$ mmole) at $10-15{ }^{\circ} \mathrm{C}$. Reaction mass was heated to $40{ }^{\circ} \mathrm{C}$ and stirred for $4 \mathrm{~h}$. Reaction progress was monitored by TLC (20\% ethyl acetate in petroleum ether, $\left.\mathrm{R}_{f}=0.65\right)$. Reaction mass cooled to room temperature, concentrated under reduced pressure to remove methanol completely. To reaction mass was added water $(50 \mathrm{~mL})$ extracted using ethyl acetate $(3 \times 20 \mathrm{~mL})$. Combined organic layers dried over sodium sulfate, filtered and concentrated under reduced pressure to get orange colored solid, yield 72\%, $2.70 \mathrm{~g}$. This was used as such for the next step.

\section{Step- III: 4-(Methylthio)benzene-1,2-diamine (13)}

To a solution of $(\mathbf{1 2})(2.7 \mathrm{~g}, 14.7 \mathrm{mmole})$ in ethanol $(25.0 \mathrm{~mL})$ was added concentrated hydrochloric acid $(2.0 \mathrm{~mL})$ at $10-15{ }^{\circ} \mathrm{C}$ was stirred for $10 \mathrm{~min}$. Reaction mass warmed to room temperature was added iron $(4.1 \mathrm{~g} 73.4$ mmole), stirred at room temperature for $4 \mathrm{~h}$. Reaction progress was monitored by TLC ( $20 \%$ ethyl acetate in petroleum ether, $\left.\mathrm{R}_{f}=0.5\right)$. To the reaction mass was added ethyl acetate: water $(100 \mathrm{~mL}, 1: 1 ; \mathrm{v} / \mathrm{v})$, basify using sodium hydroxide pellets $(4.6 \mathrm{~g})$. Reaction mass was filtered through hi-flo for better separation of organic layer from aqueous. Organic layer separated dried over sodium sulfate, filtered and concentrated under reduced pressure to get brown colored solid, yield 89\%, $2.0 \mathrm{~g}$. This was used as such for the next step.

\section{Step-IV: Methyl-N-cyanocarbamate sodium salt (14)}

To a solution of cyanamide $(4.0 \mathrm{~g}, 95.2 \mathrm{mmole})$ in water $(15 \mathrm{~mL})$ was added methyl chloroformate ( $6.0 \mathrm{~g}, 63.5 \mathrm{mmole})$ and sodium hydroxide $(5.5 \mathrm{~g}, 137.5 \mathrm{mmole})$ was added simultaneously while maintaining temperature below $10{ }^{\circ} \mathrm{C}$ and $\mathrm{pH} 7-7.5$. After addition was complete $\mathrm{pH}$ was adjusted to $8-8.5$ using aqueous sodium hydroxide solution. Reaction mass was maintained for $2 \mathrm{~h}$ at $10-15^{\circ} \mathrm{C}$. Reaction mass evaporated under reduced pressure to get white solid, yield $100 \%, 11.61 \mathrm{~g}$. This reagent was used as such for next step.

\section{Step-V: Methyl [5-(methylthio)-1H-benzimidazole-2-yl]carbamate (15)}

To a solution of $(\mathbf{1 3})(2.0 \mathrm{~g}, 12.9 \mathrm{mmole})$ in mixture of acetone $(20 \mathrm{~mL})$ and water $(2 \mathrm{~mL})$ was added concentrated hydrochloric acid $(5 \mathrm{~mL})$ at room temperature. To the reaction mass was added (11) (11.61 g, $95.1 \mathrm{mmole})$ at room temperature. Reaction mass was then heated at $80-85^{\circ} \mathrm{C}$, maintained for $3 \mathrm{~h}$. Reaction progress was monitored by TLC ( $80 \%$ ethyl acetate in petroleum ether, $\mathrm{R}_{f}=0.7$ ). Reaction mass was acidified using concentrated hydrochloric acid to $\mathrm{pH}$ 4.0-4.5. Solid obtained was filtered, washed with water $(50 \mathrm{~mL})$ and ethyl acetate $(10 \mathrm{~mL})$. Blue colored solid was dried under reduced pressure, yield 52\%, $1.60 \mathrm{~g}$, HPLC purity $92.74 \%, \mathrm{mp} 147.2{ }^{\circ} \mathrm{C}$. IR $\left(\mathrm{V}_{\max }, \mathrm{cm}^{-1}\right)$ 3323-3200 (N-H), 2948-2800 (aliphatic-H), 1737 amide N-H), 1627-1604 (C=C stretching), 1249 (C-S stretching), 800, 765, 748 (C-H bending). MS: $\mathrm{m} / z 238[\mathrm{M}+\mathrm{H}]^{+}$, $260[\mathrm{M}+\mathrm{Na}], 236[\mathrm{M}-\mathrm{H}]^{-} .{ }^{1} \mathrm{H}-\mathrm{NMR}\left(\mathrm{DMSO}_{6}\right)$ : $7.30-7.25\left(1 \mathrm{H}_{\text {arom }}, \mathrm{dd}\right), 7.45\left(1 \mathrm{H}_{\text {arom }}\right.$, d), 7.55-7.5 $\left(1 \mathrm{H}_{\text {arom }}, \mathrm{d}\right), 3.8\left(3 \mathrm{H}, \mathrm{s},-\mathrm{OCH}_{3}\right), 2.5\left(3 \mathrm{H}, \mathrm{s},-\mathrm{SCH}_{3}\right)$.

\section{Results and Discussion}

All six Albendazole impurities as per EP monograph are needed to synthesize individually in pure form. Scope of current work was to synthesize these impurities. Albendazole impurity A; 5-(propylthio)-1H-benzimidazol-2-amine (6), impurity B; methyl [5-(propylsulfinyl)- $1 \mathrm{H}$ benzimidazol-2-yl]carbamate (7), impurity C; methyl [5-(propylsulfonyl)-1H-benzimidazol 2-yl]carbamate (8) and impurity D; 5-(propylsulfonyl)-1H-benzimidazol-2-amine (9) are degradation or metabolic impurities (Figure 1). 


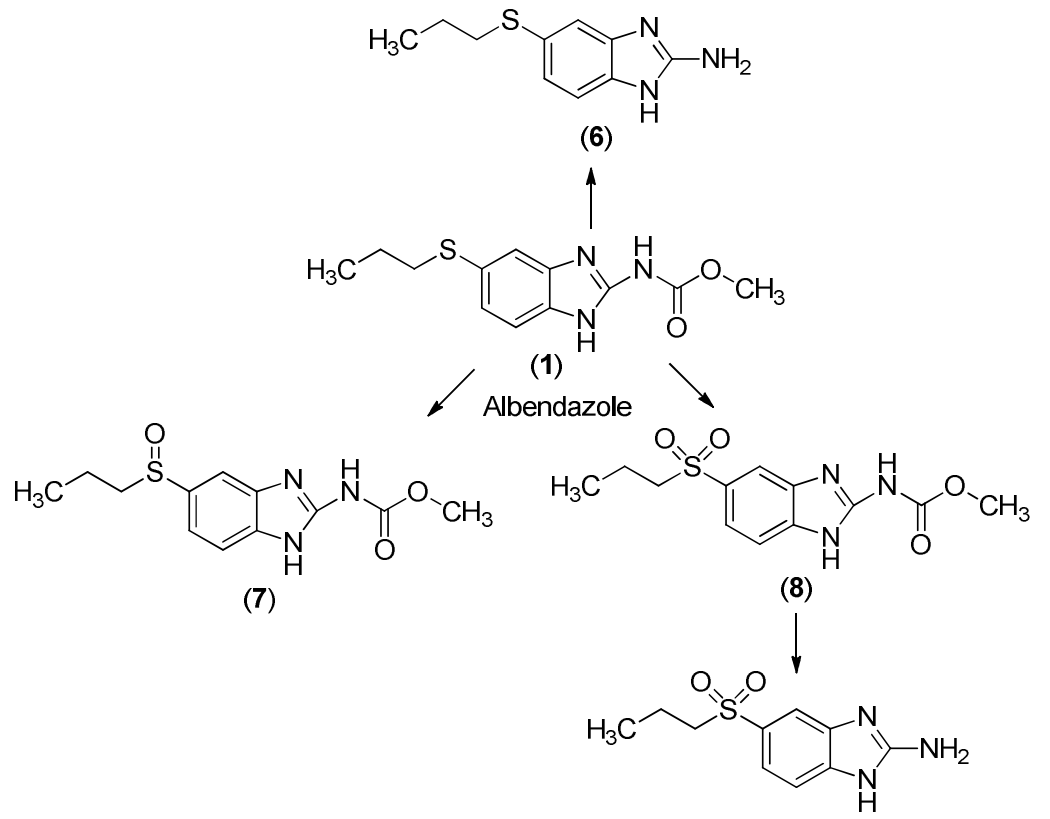

(9)

Figure 1. Degradation or metabolic impurities

Albendazole (1) was synthesized as reported in literature ${ }^{8}$ from 2-nitroaniline (2) in four steps, first step involves thiocyanation followed by $S$-alkylation using $n$-propyl bromide under basic condition gives 4-(propylthio)-2-nitroaniline (4). Reduction of compound (4) using sodium hydrosulfide on treatment with methyl- $N$-cyano carbamate yields albendazole (1) (Scheme 1).

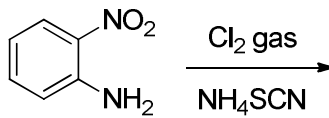

(2)<smiles>CCCBr</smiles>

(3)<smiles>CCCSc1ccc(N)c([N+](=O)[O-])c1</smiles>

(4) $\downarrow \underset{\triangle}{\text { aq. } \mathrm{NaSH}}$<smiles>CCCSc1ccc2[nH]c(NC(=O)OC)nc2c1</smiles><smiles>CCCSc1ccc(N)c(N)c1</smiles>

(1)

Albendazole

\section{Scheme 1}

Albendazole was subjected to hydrolysis condition using aqueous sulfuric acid at reflux temperature to get corresponding amine as impurity A (EP); 5-(propyl- thio)- $1 \mathrm{H}$ benzimidazol-2-amine (6) (Scheme 2).<smiles>CCCSc1ccc2[nH]c(NC(=O)OC)nc2c1</smiles>

(1)<smiles>CCCSc1ccc2[nH]c(N)nc2c1</smiles>

(6)

\section{Scheme 2}


Albendazole rapidly converted in the liver to the primary metabolite, albendazole sulfoxide, which is further metabolized to albendazole sulfone and other primary oxidative metabolites that have been identified in human urine ${ }^{11,12}$. Albendazole sulfoxide and sulfone, oxidative degradation impurities or oxidative metabolites are listed as impurity B; methyl [5-(propylsulfinyl)- 1H-benzimidazol-2-yl]carbamate (7) and impurity C; methyl [5-(propylsulfonyl)-1H- benzimidazol-2-yl]carbamate (8) respectively in EP monograph. Albendazole sulfoxide was obtained by oxidation of albendazole using Oxone in aqueous methanol and sulfone using oxidation with meta-chloroperbenzoic acid ( $m$-CPBA) in methylene chloride (Scheme 3).<smiles>CCCSc1ccc2[nH]c(NC(=O)OC)nc2c1</smiles>

(1)<smiles>CCCCOc1ccccc1</smiles>

(8)<smiles>CCCS(=O)c1ccc2[nH]c(NC(=O)OCCOc3ccccc3)nc2c1</smiles>

(7)

\section{Scheme 3}

Methyl [5-(propylsulfonyl)-1H-benzimidazol-2-yl]carbamate (8) was obtained from Albendazole oxidation as impurity $\mathrm{C}$ was hydrolyzed under acidic condition using aqueous sulfuric acid at high temperature to get corresponding amine as impurity $\mathrm{D}$, 5-(propylsulfonyl)- $1 H$-benzimidazol-2-amine (9) (Scheme 4).<smiles>CCCS(=O)(=O)c1ccc2[nH]c(NC(=O)OC)nc2c1</smiles>

(8)

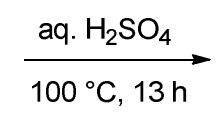

\section{Scheme 4}<smiles>CCCS(=O)(=O)c1ccc2[nH]c(N)nc2c1</smiles>

(9)

Albendazole impurity E, methyl $1 H$-benzoimidazol-2-yl)carbamate (11) and impurity F, methyl [5-(methylthio)-1H- benzimidazol-2-yl]carbamate (15) are process related impurities. Formation of impurity E (11) in API synthesis came from unreacted 2-nitroaniline (2) in thiocyanation reaction carried forward till end. Impurity F (15) is side reaction due to $S$-methylation instead of $n$-propylation in API synthesis. This may be because of methyl halide contamination in $n$-propyl halide.

Albendazole impurity E, methyl $1 H$-benzoimidazol-2-yl)carbamate (11) is synthesized by reacting 2-aminobenzimidazole (10) with methyl chloroformate in basic condition (Scheme 5).<smiles>Nc1nc2ccccc2[nH]1</smiles>

(10)<smiles>CCOC(=O)OCC(C)(C)OCC</smiles>

(11)

Scheme 5 
Methylthio analog of Albendazole is impurity F, methyl [5-(methylthio)- $1 \mathrm{H}$ benzimidazol-2-yl]carbamate (15) is synthesized in four steps from 2-nitroaniline (2). First step involves thiocyanation of 2-nitroaniline (2) yields 2-nitro-4-thiocyanoaniline (3) which was then reacted with methyl iodide to give corresponding methylthio derivative, 4(methylthio)- 2-nitroaniline (12). 4-(methylthio)-2-nitroaniline (12) was reduced using $\mathrm{Fe} / \mathrm{HCl}$ to diamine compound, 4-(methylthio)benzene-1,2-diamine (13) which further reacted with methyl-n-cyanocarbamate sodium salt (14) to give methyl [5-(methyl- thio)$1 H$ - benzimidazol-2-yl]carbamate (15) (Scheme 6). Methyl- $N$-cyano- carbamate sodium salt (14) can be obtained easily by reacting cyanamide with methyl chloroformate in presence of aqueous sodium hydroxide.

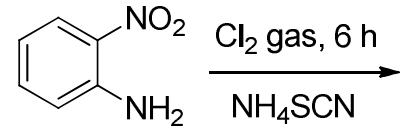

(2)<smiles>N#Sc1ccc(N)c([N+](=O)[O-])c1</smiles>

(3)<smiles>CO[N+](=O)[O-]</smiles>

$40^{\circ} \mathrm{C}, 4 \mathrm{~h}$<smiles>CSc1ccc(N)c([N+](=O)[O-])c1</smiles>

(12)

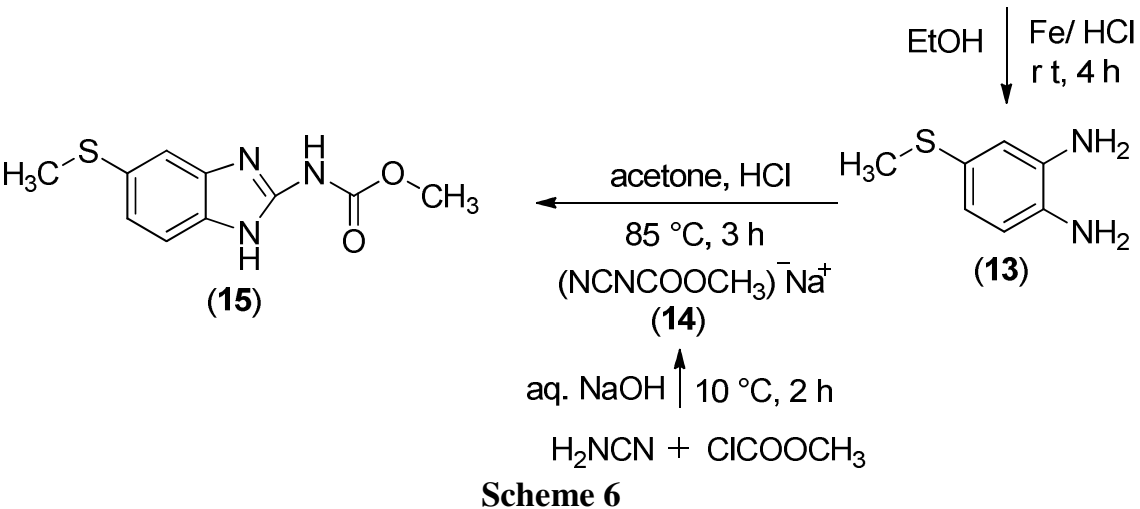

\section{Acknowledgement}

The authors express their thanks to Dr. Nitin Borkar (CEO), VerGo Pharma Research Lab. Pvt. Ltd. and Dr. S. K. Paknikar (Adviser) for their constant encouragements. Authors are also thankful to Alex Daniel and Team in the Analytical Division of VerGo Pharma Research Lab. Pvt. Ltd. for providing analytical and spectral data.

\section{References}

1. Townsend L B and Wise D S, Parasitol Today, 1990, 6(4), 107-112; DOI:10.1016/0169-4758(90)90226-T

2. Ehteda A, Galettis P, Chu S.W, Pillai K and Morris D L, Anticancer Res., 2012, 32(9), 3659-3666.

3. Králová V, Hanušová V, Staňková $\mathrm{P}$, Knoppová $\mathrm{K}$, Čáňová $\mathrm{K}$ and Skálová L, Anticancer Drugs, 2013, 24, 911-919; DOI:10.1097/CAD.0b013e3283648c69

4. ICH Harmonised Tripartite Guideline-Impurities: Guideline for residual solvents Text and Methodology Q3C (R5), Current Step 4 version, London, 2011.

5. Ahuja S, Impurities Evaluation of Pharmaceuticals, Marcel Dekker, New York 1998, 1.

6. Hazelton J C, Iddon B, Suschitzky H and Woolley L H, Tetrahedron, 1995, 51(39), 10771-10794; DOI:10.1016/0040-4020(95)00642-L

7. Walter T J, US 4,152,522, 1979. 
8. Naithani S, Natikar R D, Rane R A and Verma S, WO2012/70069 A2. http://www.google.com/patents/WO2012149438A1?cl=en

9. Vázquez G N, Yépez L, Campos A H, Tapia A, Luis F H, Cedillo R, González J, Fernández A M, Grueiro M M and Castillo R, Bioorg Med Chem., 2003, 11(21), 4615-4622; DOI:10.1016/S0968-0896(03)00497-8

10. Shahare M B, Kadam V J, Jagdale D M, Gandhi P S and Gaikwad P L, Int J Res Pharm Chem., 2012, 2(1), 132.

11. Mahler G, Davyt D, Gordon S, Incerti M, Núñez I, Pezaroglo H, Scarone L, Serra G, Silvera $\mathrm{M}$ and Manta E, J Chem Educ., 2008, 85(12), 1652; DOI:10.1021/ed085p1652

12. Romvári Z, Fekete J, Kemény S, Pokol G, Gebefügi I and Kettrup A, Chromatographia, 1998, 48(11), 777-784; DOI:10.1007/BF02467647 